\title{
Effects of the Menstrual Cycle and Acute Aerobic Exercise on Cytokine Levels
}

\author{
Harumi Hayashida ${ }^{1,2}$, Mayura Shimura ${ }^{3}$, Kaoru Sugama ${ }^{2,4}$, Kazue Kanda $^{2}$ and Katsuhiko Suzuki ${ }^{*}$ \\ ${ }^{1}$ Faculty of Health Promotional Sciences, Tokoha University, Japan \\ 2Institute for Nanoscience \& Nanotechnology, Waseda University, Japan \\ ${ }^{3}$ Faculty of Health Sciences, Health Science University, Japan \\ ${ }^{4}$ Division of Cell Regeneration and Transplantation, Department of Functional Morphology, Nihon University School of Medicine, Japan \\ ${ }^{5}$ Faculty of Sport Sciences, Waseda University, Japan
}

\begin{abstract}
Objective: The purposes of this study were: 1) to investigate the effects of menstrual cycle on the basal levels of salivary cytokines (interleukin (IL)-6 and IL-8) in young healthy women; 2) to examine the influences of prolonged aerobic exercise at ventilatory threshold (VT) on salivary and circulating IL-6 and IL-8 concentrations during menstrual phase.

Methods: Eight healthy women participated in three visits (saliva sampling during menstrual, follicular and luteal phases) and two trials (exercise and resting trials during a separate menstrual phase). The exercise trial involved a 60 - min cycling at VT and the resting trial involved resting for $90 \mathrm{~min}$. Saliva and blood samples were taken at before, immediately after and $30 \mathrm{~min}$ post-exercise and equivalent times during the resting trial.

Results: Salivary IL-6 level was higher during the follicular phase than the luteal phase at rest. Salivary IL- 8 immediately post-exercise was higher than pre-exercise, and circulating IL-6 was significantly increased from preexercise to immediately post-exercise and 30 min post exercise.

Conclusion: The results showed that salivary cytokines are influenced by menstrual cycle and prolonged exercise during menstrual phase. However the levels of IL-6 and IL-8 in saliva do not closely reflect those in plasma, and therefore might reflect a local level of inflammation.
\end{abstract}

Keywords: Menstrual cycle; Inflammation; Ventilatory threshold; Endurance exercise; Interleukin-6; Interleukin-8

\section{Introduction}

It has been shown that physical and psychological stresses are associated with the phases of the menstrual cycle [1,2]. Only few studies have examined the relationship between stress level and inflammation status during different phases of menstrual cycle using biological stress markers and cytokines such as cortisol and interleukin (IL)-6, respectively [1-3]. Cortisol is a well-known biological stress marker whereas IL- 6 is a multifunctional cytokine that is secreted by endometrial cells, immune cells and muscle cells [4,5] and is a major factor in the regulation of the inflammatory process, immune response, metabolism and the acute phase response [5,6]. In the previous studies, healthy women with a normal menstrual cycle demonstrated higher serum cortisol levels during luteal phase compared with the follicular phase [1] yet plasma IL-6 levels were higher during the follicular phase compared with the luteal phase [2].

On the other hand, other studies report similar serum IL-6 levels between follicular and luteal phases [3]. To date, no studies have examined markers of inflammation specifically during the menstrual phase of the cycle. This is because the menstrual phase has been considered as a part of the follicular phase [2,3]. Female sex hormones, especially progesterone, influence immune responses by decreasing basal IL-6 levels [2]. Moreover, menstruation, initiated by progesterone withdrawal, is highly inflammatory in nature and involves feedback loops that enhance inflammatory pathways [7]. Therefore, it is important to specifically focus on menstrual phase as a distinct phase.

Recently, saliva sampling has received attention as an alternative to blood sampling to measure stress markers [8-11]. Saliva collection has the advantage over blood sampling in that it is a non-invasive method of sample collection which does not require any specialized training. However, the influence of menstrual cycle on stress markers using saliva has received comparatively little attention. In healthy women with a normal menstrual cycle, saliva samples were taken every evening to compare cortisol levels among menstrual, follicular, ovulatory, luteal and premenstrual phases and the analysis revealed that salivary cortisol level did not differ across the phases [12]. In another study, female participants provided a saliva sample directly after awakening and 30, 45 and $60 \mathrm{~min}$ thereafter to measure cortisol levels during the menstrual phase, follicular phase, ovulation and luteal phase. The results showed that there was a significant interaction between time and menstrual cycle and the net increase in cortisol level was larger during the ovulation than during the menstrual, follicular and luteal phases $[13,14]$. It is well known that saliva cortisol exhibits diurnal variations with concentrations highest in the morning and this may account for the disparities in the apparent influence of menstrual phase on cortisol levels between these studies.

Several studies have examined salivary IL-6 and/or IL-8 concentrations in relation to systemic or local disease conditions including oral cancers [15,16] and Sjögren's syndrome [15], an autoimmune disease that affects mucosal exocrine functions. Other studies have assessed the relationships between salivary IL-6 levels and

*Corresponding author: Katsuhiko Suzuki, Professor, Faculty of Sport Sciences, Waseda University, \#2-579-15 Mikajima, Tokorozawa, Saitama 359-1192, Japan, Tel: +81-4-2947-6898; E-mail: katsu.suzu@waseda.jp

Received November 17, 2015; Accepted December 17, 2015; Published December 23, 2015

Citation: Hayashida H, Shimura M, Sugama K, Kanda K, Suzuki K (2015) Effects of the Menstrual Cycle and Acute Aerobic Exercise on Cytokine Levels. J Sports Med Doping Stud 6: 173. doi:10.4172/2161-0673.1000173

Copyright: (c) 2015 Hayashida $\mathrm{H}$ et al. This is an open-access article distributed under the terms of the Creative Commons Attribution License, which permits unrestricted use, distribution, and reproduction in any medium, provided the original author and source are credited. 
psychosocial factors in middle aged people [17] and in post-menopausal women [18]. Therefore, there is a lack of information regarding salivary IL- 6 and IL- 8 concentrations on resting healthy women during different phases of menstrual cycle. In addition, previous studies reported that salivary and circulating IL-6 levels do not relate to each other at rest $[15,17,18]$ and at post-exercise [10]. Such findings need to be confirmed in healthy women at different timings of menstrual cycle to demonstrate whether salivary sampling can be employed as an alternative to blood sampling for an examination of inflammation status.

Moderate intensity aerobic exercise is important for maintaining or promoting physical fitness. However, exercise has been shown to induce an inflammatory response and estrogen is associated with an attenuation of the response [19]. Moreover, the secretion of estrogen changes during the menstrual cycle yet reported circulating inflammatory responses to exercise during the different phases of the menstrual cycle are equivocal [20], which may reflect differences in participant fitness, oral contraceptive use and differing exercise protocols used. Considering women's health and safety and the effectiveness of exercise, it is important to assess the influence of exercise or changes in the stress and inflammatory markers during the menstrual cycle [21]. Only one study has examined an influence of prolonged moderate intensity exercise on salivary IL-6 and reported that salivary IL- 6 level was higher at $30 \mathrm{~min}$ post treadmill running ( $1 \mathrm{~h}$ at $65 \% \mathrm{VO}_{2}$ peak) compared to the baseline level during follicular and luteal phases in females [22]. However, the magnitude of increases did not differ between the follicular and the luteal phases. Hence, an influence of exercise on inflammation status during menstrual phase is still unclear.

Therefore, the aims of this study were: 1) to investigate the effects of menstrual cycle on the basal levels of salivary cytokines (IL-6 and IL-8) in young healthy women; 2) to examine the influences of prolonged aerobic exercise at ventilatory threshold (VT) on salivary and circulating IL-6 and IL-8 levels during menstrual phase. It was hypothesised that: 1) basal salivary cytokine levels would be affected by menstrual cycle; and 2) salivary cytokine levels would be affected by acute aerobic exercise at VT.

\section{Materials and Methods}

\section{Subjects}

Eight healthy young women enrolled in this study (age: $20.5 \pm 0.8$ years (means \pm standard deviation (SD), body mass: $48.3 \pm 5.9 \mathrm{~kg}$, height: $155.4 \pm 4.9 \mathrm{~cm}, \mathrm{BMI}: 20.0 \pm 2.2 \mathrm{~kg} / \mathrm{m}^{2}$, estimated body fat: 23.7 $\pm 4.0 \%$ and $\mathrm{VO}_{2}$ peak: $1790 \pm 281 \mathrm{ml} / \mathrm{min}$ ). None of them were taking any medications including oral contraceptives or dietary supplements. Their menstrual cycle was regular and there were no menstrual cyclerelated disorders (e.g., premenstrual syndrome, dysmenorrhea) for at least one year. All subjects were recreationally active and exercised for less than $1 \mathrm{~h}$ /week at moderate intensity (e.g., jogging, recreational volleyball and basketball). Written informed consent was obtained from all participants and they were free to withdraw from the study at any time. The experimental procedure was approved by the Research Ethics Committee at Waseda University.

\section{Experimental design}

The subjects attended the laboratory on seven separate occasions for two preliminary trials, three short visits and two main trials. Two preliminary trials were arranged for orientation, to familiarize the subjects with the experimental procedures and to determine their VT, height, body mass, and estimated body fat (OMRON BODY FAT MONITOR HBF-903, Omron Healthcare, Kyoto, Japan). The visits took place during the menstrual phase (day 1-4 from the start of menstrual flow), follicular phase (day 7-11 after the start of menstrual flow) and mid-luteal phase (5-9 days before estimated date of the next menstrual phase) and main trials were conducted during menstrual phase. The subjects refrained from performing any strenuous physical activity and drinking alcohol for at least $24 \mathrm{~h}$ prior to the testing trials, and consumed a small meal at 12:00 pm before the trials. To control for diurnal variations in the hormones, all trials were performed at the same time of day from 2:00 to 3:00 pm.

\section{Determination of VT}

In a preliminary session, peak oxygen uptake ( $\mathrm{VO}_{2}$ peak) was estimated using a continuous incremental exercise test performed on an electromagnetically braked cycle ergometer (AEROBIKE 75XL, Combi, Shinagawa, Tokyo, Japan). Oxygen consumption, ventilation and heart rate were measured during an incremental cycle ergometry test (2 Watts increase every 10 seconds, starting at 20 Watts) with a bicycle ergometer. Ventilation was measured and gas analysis was performed during the test on a breath-by-breath basis (AEROMONITOR AE300S, Minato, Yodogawa, Osaka, Japan). VT was determined by the modified $\mathrm{V}$-slope method, which is from a plot of $\mathrm{VCO}_{2}$ as a function of $\mathrm{VO}_{2}[23]$.

\section{Visits and main trials}

For the three visits, the subjects reported to the laboratory in a rested state during the menstrual phase, follicular phase and mid-luteal phase to provide saliva and blood samples. For the main trials, subjects reported to the laboratory at 2:00 pm for the exercise and control trials in a separate menstrual phase. In the exercise trial, subjects cycled for $60 \mathrm{~min}$ at the predetermined VT from $2: 30 \mathrm{pm}$ and they seated quietly for $30 \mathrm{~min}$ after the cycling. Saliva and blood samples were taken at pre-, immediately post- and $30 \mathrm{~min}$ post-exercise. Subjects drank water ad libitum during each trial to maintain hydration status. Heart rate was measured using a bedside monitor (BSM-2401, Nihonkohden, Shinjuku, Tokyo, Japan). In the control trial, subjects remained seated quietly for $90 \mathrm{~min}$ from $2: 30 \mathrm{pm}$ and saliva specimens were collected at 0,60 and $90 \mathrm{~min}$ (i.e. at the same times of day as in the exercise trial).

\section{Sample collection and analyses}

Each participant was instructed to empty her mouth of pre-existing saliva before sampling. One and a half $\mathrm{ml}$ of unstimulated saliva was collected before blood sampling by passive drool for determination of hormone and cytokine levels. Saliva samples were centrifuged at 3,000 $\mathrm{rpm}$ for $15 \mathrm{~min}$ at room temperature and stored at $-30^{\circ} \mathrm{C}$ until the day of analyses. All salivary samples were centrifuged again on the day of the assay to remove particulate matter.

Capillary blood samples were obtained from the fingertip into at least three heparinized capillary tubes for hematocrit measurement. The capillary tubes were immediately centrifuged at $12,000 \mathrm{rpm}$ for $5 \mathrm{~min}$ at room temperature. Hematocrit was measured to confirm changes in plasma volume and hydration status. After the hematocrit measurement, the plasma was put into microtubes and stored at $-30^{\circ} \mathrm{C}$ until the day of assays.

To confirm the menstrual cycle, the hormonal status was determined by measuring salivary $17 \beta$-estradiol and progesterone concentrations [24]. The salivary 17 $\beta$-estradiol, progesterone, cortisol and Dehydroepiandrosterone (DHEA) concentrations were measured using high sensitivity enzyme immunoassay (EIA) kits (Salimetrics LLC, Sate College, PA, USA). The intra-assay coefficients of variation for $17 \beta$-estradiol, progesterone, cortisol and DHEA were $8.1 \%, 6.2 \%$, 
3.6 and $5.5 \%$, respectively. The salivary amylase activity was measured using an enzymatic method (AMYLASE MONITOR, Nipro Co., Osaka, Japan) [25]. The saliva concentrations of IL- $1 \beta$ and TNF- $\alpha$ were measured using Quantikine high sensitivity (HS) enzyme-linked immunosorbent assay (ELISA) kits (R\&D Systems, Minneapolis, MN, USA). The intra-assay coefficients of variation for IL- $1 \beta$ and TNF- $\alpha$ were 4.4 and $8.5 \%$, respectively. The saliva concentrations of protein were measured by the Bradford method using a protein assay kit purchased from Bio-Rad (Hercules, CA, USA) [26]. Resting and exercise-induced salivary and plasma concentrations of IL- 6 were measured using Quantikine HS ELISA kits (R\&D Systems, Minneapolis, MN, USA) and IL-8 was measured using OptEIA kits (Becton Dickinson Bioscience, San Diego, CA, USA). The intra-assay coefficients of variation for IL-6 and IL-8 were 7.8 and 5.5\%, respectively, and inter-assay coefficients of variation were 14.2 and $10.0 \%$, respectively. These measurements were performed with a microplate reader (VERSAMAX, Molecular Devices Inc. Sunnyvale, CA, USA). Plasma and saliva samples from each individual subject were tested in the same assay run.

\section{Statistical analyses}

A one-way analysis of variance with repeated measures was used to assess the differences in basal salivary hormone and cytokine levels among the menstrual, follicular and luteal phases. A two-way (trial $\mathrm{x}$ time) analysis of variance with repeated measures was used to examine the differences in cytokine levels. When a main effect was observed, post hoc tests using Bonferroni adjustment was employed for multiple comparisons. Normality plots with tests (Shapiro-Wilks) were performed and log transformed before statistical analysis when data were not normally distributed. After log transformation, data were investigated again and considered to be normally distributed. Effect sizes $\left(\eta^{2}\right)$ were calculated using guidelines of Cohen [27], and were considered as follows: $0.01=$ small effect; $0.06=$ moderate effect; and $0.14=$ large effect. Data are presented as mean \pm SD. Spearman's correlation coefficients were employed to assess the relationships between the salivary and plasma values. Statistical significance was set at $\mathrm{P}<0.05$. Statistical analyses were performed using PASW Statistics 18 software (SPSS, Chicago, IL, USA).

\section{Results}

\section{Menstrual cycle effects}

The descriptive data for resting salivary hormones and plasma cytokines during each menstrual phase are presented in Table 1. Salivary $17 \beta$-estradiol concentration was not different between menstrual and follicular phases, however, the concentration increased by $41 \%$ from follicular phase to luteal phase (Table 1). Salivary progesterone concentration was increased by $156 \%$ from menstrual phase to luteal phase, and $177 \%$ from follicular phase to luteal phase (Table 1).

Salivary IL-6 concentration significantly differed between different phases of menstrual cycle $\left(\mathrm{P}=0.023, \eta^{2}=0.42\right)$. When Bonferroni adjustment was conducted, salivary IL- 6 concentration was significantly higher during the follicular phase compared with the luteal phase $(\mathrm{P}$ $=0.036$ ) (Figure 1). Salivary TNF- $\alpha$, IL- $1 \beta$ and IL- 8 concentrations tended to decrease from the menstrual phase to the luteal phase but the concentrations were not significantly affected by menstrual cycle (TNF- $\alpha: \mathrm{P}=0.059, \eta^{2}=0.33, \mathrm{IL}-1 \beta: \mathrm{P}=0.474, \eta^{2}=0.10, \mathrm{IL}-8: \mathrm{P}=0.256$, $\left.\eta^{2}=0.18\right)$ (Figure 1).

Similarly, plasma IL-6 and IL-8 concentrations tended to decrease from the menstrual phase to the other phases but these were not significantly affected by menstrual cycle (IL-6: $P=0.153, \eta^{2}=0.18$, IL-

\begin{tabular}{|c|c|c|c|}
\hline & Menstrual & Follicular & Luteal \\
\hline \multicolumn{4}{|l|}{ Salivary hormone } \\
\hline $17 \beta$-estradiol (pg/ml) & $2.43 \pm 0.70$ & $2.51 \pm 0.60$ & $3.53 \pm 1.18$ \\
\hline Progesterone $(\mathrm{pg} / \mathrm{ml})$ & $105.21 \pm 49.21$ & $97.23 \pm 37.30$ & $269.56 \pm 145.35$ \\
\hline \multicolumn{4}{|l|}{$\begin{array}{l}\text { Salivary stress } \\
\text { marker }\end{array}$} \\
\hline Cortisol (pg/ml) & $0.19 \pm 0.13$ & $0.23 \pm 0.14$ & $0.20 \pm 0.11$ \\
\hline DHEA (pg/ml) & $174.60 \pm 115.99$ & $258.68 \pm 282.79$ & $149.19 \pm 55.29$ \\
\hline $\begin{array}{l}\text { Amylase activity } \\
\text { (KIU/L) }\end{array}$ & $67.50 \pm 52.37$ & $60.50 \pm 33.95$ & $60.13 \pm 61.33$ \\
\hline \multicolumn{4}{|l|}{ Plasma cytokine } \\
\hline IL-6 (pg/ml) & $1.23 \pm 0.90$ & $0.59 \pm 0.34$ & $0.65 \pm 0.45$ \\
\hline IL-8 (pg/ml) & $5.38 \pm 2.65$ & $3.35 \pm 2.10$ & $3.89 \pm 1.86$ \\
\hline
\end{tabular}

Table 1: Salivary hormone, stress marker and plasma cytokine data at baseline with regard to menstrual cycle phases. Data are presented as means $\pm S D(n=8)$. DHEA: Dehydroepiandrosterone, IL-6: Interleukin-6, IL-8: Interleukin-8. * $\mathrm{P}<0.05$ follicular versus luteal phase; ${ }^{P}<0.05$ menstrual versus luteal phase
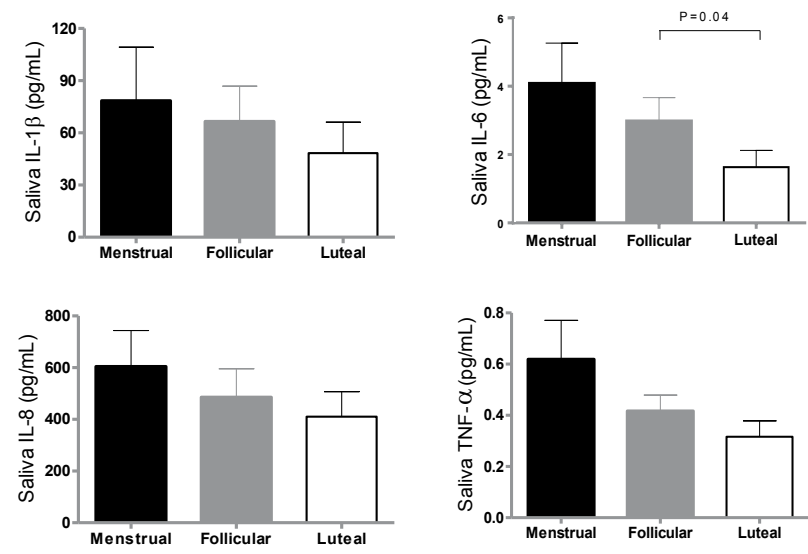

Figure 1: Salivary inflammatory cytokine data at baseline with regard to menstrual cycle phases. Data are presented as means \pm SE $(n=8)$. IL-1 Interleukin-1 $\beta$, IL-6: Interleukin-6, IL-8: Interleukin-8, TNF- $\alpha$ : Tumor necrosis factor- $\alpha$

8: $\left.\mathrm{P}=0.180, \eta^{2}=0.16\right)($ Table 1$)$. There were no significant relationships between saliva and plasma IL- 6 concentrations and between saliva and plasma IL-8 concentrations.

Salivary cortisol, DHEA concentrations and salivary amylase activity at rest were unaffected by the menstrual cycle (Table 1).

\section{Exercise effects}

During the 60 -min cycling at VT, subjects cycled at $63.3 \pm 9.3$ Watts, corresponding to $78.5 \pm 15.4 \%$ HRmax (using Karvonen formula). The changes in salivary and plasma IL- 6 and IL- 8 concentrations during the exercise and control trials are presented in Figure 2. A significant interaction (trial by time) was found in plasma IL-6 $(\mathrm{P}=0.001)$. Immediately and 30-min post-exercise plasma IL-6 concentrations were significantly higher than that of pre-exercise (pre: $0.92 \pm 1.03 \mathrm{pg} /$ $\mathrm{mL}$, immediately post-exercise: $1.85 \pm 1.23 \mathrm{pg} / \mathrm{mL}, \mathrm{P}=0.013,30 \mathrm{~min}$ post-exercise: $2.38 \pm 2.43 \mathrm{pg} / \mathrm{mL}, \mathrm{P}=0.001$ ) (Figure 2). No significant time and trial effects were found in plasma IL-8, salivary IL-6 and protein. However, a significant interaction (trial by time) was found in salivary IL-8 $(\mathrm{P}=0.004)$. Immediately post-exercise salivary IL-8 concentration was significantly higher than that of pre-exercise $(\mathrm{P}=$ 

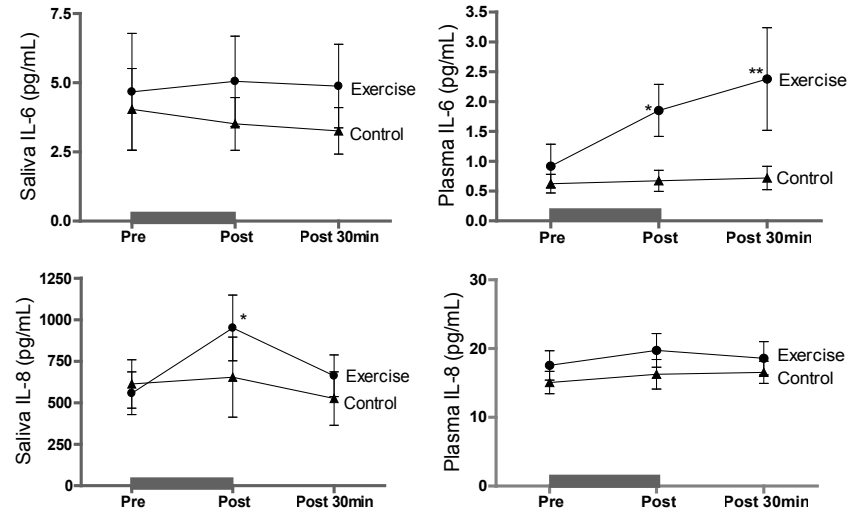

Figure 2: Changes in IL-6 and IL-8 levels in saliva and plasma during cycling at ventilator threshold $(\mathrm{VT})$ and the control trial $(n=8)$. The data given refer to exercise during the menstrual period, since, as shown in Figure 1, interleukin levels vary throughout the menstrual cycle. Data are presented as means $\pm \mathrm{SE}$ IL-6: Interleukin-6, IL-8: Interleukin-8. ${ }^{*} \mathrm{P}<0.05$ versus pre-exercise, ${ }^{* *} \mathrm{P}<$ 0.001 versus pre-exercise

0.019) (Figure 2). No significant relationships were found between the concentrations of saliva and plasma IL-6 and IL-8 (IL-6: $r=0.366, \mathrm{P}=$ $0.08, \mathrm{IL}-8: \mathrm{r}=0.312, \mathrm{P}=0.14$ )

\section{Discussion}

The current study investigated the effects of the menstrual cycle on basal salivary stress hormone and cytokine levels, and the influences of aerobic exercise at VT on cytokine levels in healthy recreationally active women. The main findings of this study were; (1) resting salivary IL-6 concentration was significantly higher during the follicular phase compared with the luteal phase; (2) immediately post-exercise salivary IL-8 concentration was significantly higher than that of pre-exercise; (3) salivary and plasma cytokines were influenced differently by menstrual cycle and exercise.

\section{Menstrual cycle effects}

In the present study, salivary IL-6 concentration was significantly affected by menstrual cycle, whereas plasma IL- 6 concentrations were unaffected by menstrual cycle in young healthy women at rest. Our findings in plasma are in contrast to a previous study that reported menstrual cycle influences on circulating IL-6 concentrations [2].

In the current study, plasma IL-6 level showed small (1.9 fold) insignificant decreases from menstrual phase to luteal phase, but no differences were observed between the follicular phase and luteal phase. However, Angstwurm et al. [2] reported that plasma IL-6 decreased from follicular phase to luteal phase. This may be related to the timing of sample correction during the menstrual cycle. This conflicting result could be attributable to the current study having used three phases of the menstrual cycle (menstrual, follicular and luteal) as opposed to two phases (follicular and luteal) used in the study by Angstwurm et al. [2].

However, no significant relationships were found between saliva and circulating IL-6 (and IL-8) concentrations in recreationally active healthy women at rest in the current study. Previous studies agree with the current study as they reported that salivary IL- 6 level was not related to circulating IL-6 at rest and post-exercise conditions in patients and mixed gender athletes $[10,15,17,18]$. Moreover, it has been discovered that IL-6 mRNA are up regulated in salivary glands of mouse models [28] and in humans [29]. Furthermore, salivary IL-6 (and IL-8) has been used to assess immunological patterns relevant to systemic or local disease conditions and its levels were altered in patients with oral health problems $[15,16,30]$. Therefore, salivary IL-6 is possibly reflecting a local level of inflammation and as such it is understandable that saliva IL-6 concentrations are not a good refection of circulating values.

In the present study, salivary IL-6 level was the lowest when concentrations of female sex hormones ( $17 \beta$-estradiol and progesterone) were the highest (luteal phase). This is possibly because female sex hormones reduce inflammations as previous studies reported that estradiol showed an anti-inflammatory action and down regulated IL-6 in vitro $[31,32]$.

All other salivary and plasma cytokines were not significantly affected by menstrual cycle. However, salivary levels of TNF- $\alpha$, IL- $1 \beta$ and IL-8 tended to decrease between the menstrual and luteal phases. Although a significant difference was not found, moderate or large effect sizes were demonstrated for these relationships, perhaps suggesting that these markers are also worthy of future attention.

In the present study, cortisol and DHEA in saliva and salivary amylase activity did not significantly differ among the menstrual cycle phases. Generally, these hormones have been used as stress markers $[11,25,33]$. Bernstein et al. [34] investigated an association between sympathetic responsiveness and the serum concentration of IL-6 in young women. However, changes in salivary amylase activity, an index of sympathetic tone according to menstrual cycle was not observed in the current study. Therefore, salivary IL- 6 concentration at rest seems to reflect the inflammatory conditions which is influenced by menstrual cycle, but not influenced by sympathetic tone.

\section{Exercise effects}

In the present study, there were no differences in salivary IL-6 concentration among before, immediately after and 30-min post cycling at VT during menstrual phase in recreationally active women with a normal menstrual cycle. However, a previous study reported that salivary IL- 6 concentration increased after a prolonged moderate intensity exercise in sedentary women [22]. In the previous study, salivary IL-6 concentration increased significantly from baseline to $30 \mathrm{~min}$ post $1 \mathrm{~h}$ treadmill running at $65 \% \mathrm{VO}_{2}$ peak during follicular and luteal phases. Potential rationales for the different outcomes are an employment of different exercise intensity and conducting the studies during different phases of menstrual cycle. In general, $65 \%$ $\mathrm{VO}_{2}$ peak is higher than $\mathrm{VT}$ and ventilation rates increase at higher exercise intensities which may lead to increased airway inflammation. Hence, higher exercise intensities may be linked with a greater local inflammation which is reflected in an increased concentration of salivary IL-6. Moreover, resting salivary IL-6 concentration is the highest during menstrual phase compared to the other phases as observed in the current study; this may limit further elevations in saliva IL-6 concentration in response to exercise during menstrual phase.

In the current study, salivary IL-8 concentration significantly increased from pre- to post-exercise. In contrast to the salivary IL-8 concentration, circulating IL-8 concentration did not change throughout the trial. IL- 8 is a neutrophil chemotactic factor, released by immune cells and endothelial cells to recruit neutrophils to sites of tissue damage (i.e. inflammation) [35-37]. Hence, the increase in salivary IL-8 observed in the present study after exercise may also simply reflect local inflammation due to increased ventilatory rates with exercise. Currently, there are no other studies which examined an influence of exercise on cytokine levels during menstrual phase using both salivary and circulating IL-8 concentrations. Therefore, further research is required to confirm the findings from the present study. 
In conclusion, salivary cytokines (IL-6) is influenced by menstrual cycle at rest in recreationally active healthy women. Moreover, salivary IL- 6 and IL-8 responded differently to both the menstrual cycle and to exercise compared with their counterparts in plasma. Therefore saliva sampling should not be considered as a non-invasive alternative to blood sampling for examining the inflammation status in women as it may simply reflect local inflammatory events related to increased ventilatory rates.

\section{Acknowledgements}

This study was supported by a Grant-in-Aid for the Global COE "Sport Sciences for the Promotion of Active Life", Scientific Research (A) 15H0183, and the Strategic Research Foundation at Private Universities, 2015-2020 from the Ministry of Education, Culture, Sports, Science and Technology (S1511017) of Japan.

The authors would like to thank Dr. Nicolette Bishop for her help with preparing this manuscript.

\section{References}

1. Tersman Z, Collins A, Eneroth $P$ (1991) Cardiovascular responses to psychological and physiological stressors during the menstrual cycle. Psychosom Med 53: 185-197.

2. Angstwurm MW, Gärtner R, Ziegler-Heitbrock HW (1997) Cyclic plasma IL-6 levels during normal menstrual cycle. Cytokine 9: 370-374.

3. Chiu KM, Arnaud CD, Ju J, Mayes D, Bacchetti P, et al. (2000) Correlation of estradiol, parathyroid hormone, interleukin- 6 , and soluble interleukin-6 receptor during the normal menstrual cycle. Bone 26: 79-85.

4. Ray P, Ghosh SK, Zhang DH, Ray A (1997) Repression of interleukin-6 gene expression by 17 beta-estradiol: inhibition of the DNA-binding activity of the transcription factors NF-IL6 and NF-kappa B by the estrogen receptor. FEBS Lett 409: 79-85.

5. Pedersen BK, Febbraio MA (2008) Muscle as an endocrine organ: focus on muscle-derived interleukin-6. Physiol Rev 88: 1379-1406.

6. Pedersen BK (2011) Muscles and their myokines. J Exp Biol 214: 337-346

7. Evans J, Salamonsen LA (2012) Inflammation, leukocytes and menstruation. Rev Endocr Metab Disord 13: 277-288.

8. Kirschbaum C, Kudielka BM, Gaab J, Schommer NC, Hellhammer DH (1999) Impact of gender, menstrual cycle phase, and oral contraceptives on the activity of the hypothalamus-pituitary-adrenal axis. Psychosom Med 61: 154-162.

9. Minetto M, Rainoldi A, Gazzoni M, Terzolo M, Borrione P, et al. (2005) Differential responses of serum and salivary interleukin-6 to acute strenuous exercise. Eur J Appl Physiol 93: 679-686.

10. Minetto MA, Gazzoni M, Lanfranco F, Baldi M, Saba L, et al. (2007) Influence of the sample collection method on salivary interleukin-6 levels in resting and post-exercise conditions. Eur J Appl Physiol 101: 249-256.

11. Thomasson R, Baillot A, Jollin L, Lecoq AM, Amiot V, et al. (2010) Correlation between plasma and saliva adrenocortical hormones in response to submaximal exercise. J Physiol Sci 60: 435-439.

12. Brown SG, Morrison LA, Calibuso MJ, Christiansen TM (2008) The menstrual cycle and sexual behavior: relationship to eating, exercise, sleep, and health patterns. Women Health 48: 429-444.

13. Kudielka BM, Kirschbaum C (2003) Awakening cortisol responses are influenced by health status and awakening time but not by menstrual cycle phase. Psychoneuroendocrinology 28: 35-47.

14. Wolfram M, Bellingrath S, Kudielka BM (2011) The cortisol awakening response (CAR) across the female menstrual cycle. Psychoneuroendocrinology 36: 905912

15. Tishler M, Yaron I, Shirazi I, Yossipov Y, Yaron M (1999) Increased salivary interleukin-6 levels in patients with primary Sjögren's syndrome. Rheumatol Int 18: $125-127$

16. St John MA, Li Y, Zhou X, Denny P, Ho CM, et al. (2004) Interleukin 6 and interleukin 8 as potential biomarkers for oral cavity and oropharyngeal squamous cell carcinoma. Arch Otolaryngol Head Neck Surg 130: 929-935.

17. Sjögren E, Leanderson P, Kristenson M, Ernerudh J (2006) Interleukin-6 levels in relation to psychosocial factors: studies on serum, saliva, and in vitro production by blood mononuclear cells. Brain Behav Immun 20: 270-278.

18. Fernandez-Botran R, Miller JJ, Burns VE, Newton TL (2011) Correlations among inflammatory markers in plasma, saliva and oral mucosal transudate in post-menopausal women with past intimate partner violence. Brain Behav Immun 25: 314-321.

19. Kendall B, Eston R (2002) Exercise-induced muscle damage and the potential protective role of estrogen. Sports Med 32: 103-123.

20. Gillum TL, Kuennen MR, Schneider S, Moseley P (2011) A review of sex differences in immune function after aerobic exercise. Exerc Immunol Rev 17: 104-121.

21. Northoff H, Symons S, Zieker D, Schaible EV, Schäfer K, et al. (2008) Genderand menstrual phase dependent regulation of inflammatory gene expression in response to aerobic exercise. Exerc Immunol Rev 14: 86-103.

22. Ives SJ, Blegen M, Coughlin MA, Redmond J, Matthews T, et al. (2011) Salivary estradiol, interleukin- 6 production, and the relationship to substrate metabolism during exercise in females. Eur J Appl Physiol 111: 1649-1658.

23. Sue DY, Wasserman K, Moricca RB, Casaburi R (1988) Metabolic acidosis during exercise in patients with chronic obstructive pulmonary disease. Use of the V-slope method for anaerobic threshold determination. Chest 94: 931-938.

24. Gandara BK, Leresche L, Mancl L (2007) Patterns of salivary estradiol and progesterone across the menstrual cycle. Ann N Y Acad Sci 1098: 446-450.

25. Yamaguchi M, Kanemaru M, Kanemori T, Mizuno Y (2003) Flow-injection-type biosensor system for salivary amylase activity. Biosens Bioelectron 18: 835840 .

26. Bradford MM (1976) A rapid and sensitive method for the quantitation of microgram quantities of protein utilizing the principle of protein-dye binding Anal Biochem 72: 248-254.

27. Cohen JW (1988) Statistical power analysis for the behavioral sciences. Lawrence Erlbaum, Hillsdale, NJ: pp 380-403.

28. Tanda N, Ohyama H, Yamakawa M, Ericsson M, Tsuji T, et al. (1998) IL-1 beta and IL-6 in mouse parotid acinar cells: characterization of synthesis, storage, and release. Am J Physiol 274: G147-156.

29. Boumba D, Skopouli FN, Moutsopoulos HM (1995) Cytokine mRNA expression in the labial salivary gland tissues from patients with primary Sjögren's syndrome. Br J Rheumatol 34: 326-333.

30. Korostoff A, Reder L, Masood R, Sinha UK (2011) The role of salivary cytokine biomarkers in tongue cancer invasion and mortality. Oral Oncol 47: 282-287.

31. Pottratz ST, Bellido T, Mocharla H, Crabb D, Manolagas SC (1994) 17 beta-Estradiol inhibits expression of human interleukin-6 promoter-reporter constructs by a receptor-dependent mechanism. J Clin Invest 93: 944-950.

32. Kovacs EJ, Plackett TP, Witte PL (2004) Estrogen replacement, aging, and cellmediated immunity after injury. J Leukoc Biol 76: 36-41.

33. Cadore E, Lhullier F, Brentano M, Silva E, Ambrosini M, et al. (2008) Correlations between serum and salivary hormonal concentrations in response to resistance exercise. J Sports Sci 26: 1067-1072.

34. Bernstein IM, Damron D, Schonberg AL, Shapiro R (2009) The relationship of plasma volume, sympathetic tone, and proinflammatory cytokines in young healthy nonpregnant women. Reprod Sci 16: 980-985

35. Suzuki K, Totsuka M, Nakaji S, Yamada M, Kudoh S, Liu Q (1999) Endurance exercise causes interaction among stress hormones, cytokines, neutrophil dynamics, and muscle damage. J Appl Physiol 87: 1360-1367.

36. Suzuki K, Nakaji S, Yamada M, Totsuka M, Sato K, Sugawara K (2002) Systemic inflammatory response to exhaustive exercise: cytokine kinetics. Exer Immunol Rev 8: 6-48

37. Suzuki K, Nakaji S, Yamada M, Liu Q, Kurakake S, et al. (2003) Impact of competitive marathon race on systemic cytokine and neutrophil responses. Med Sci Sports Exer 35: 348-355 